\title{
The Future of Long-Term Monitoring Following Catheter and Surgical Intervention for Atrial Fibrillation
}

\author{
Graham Lohrmann ${ }^{1}$ and Rod Passman ${ }^{1}$ \\ ${ }^{1}$ Northwestern University Feinberg School of Medicine
}

December 7, 2020

\begin{abstract}
Monitoring following catheter or surgical ablation for atrial fibrillation (AF) is an essential tool used to assess outcomes for research purposes and help guide clinical decision making. The most commonly used methods to monitor for post-intervention AF include a variety of ambulatory external electrocardiogram (ECG) monitors, cardiac implantable electronic devices (CIED), and more recently, direct to consumer digital health technologies. The traditional metric of ablation success, recurrence $>$ 30 seconds at 1 year, is below the detection capabilities of almost all monitoring techniques yet still undervalues the efficacy of $\mathrm{AF}$ interventions. Measures of AF burden reduction and duration give a more complete assessment of the impact of $\mathrm{AF}$ surgeries and ablation. As it is increasingly being recognized that AF burden and duration is related to stroke risk, long-term, inexpensive, non-invasive monitoring methods are needed. Smart phones and watches with AF-detecting capabilities, which are increasingly being used by the majority of US adults, have emerged as viable options to achieve this goal, shifting the paradigm of AF monitoring to a more patient centered approach.
\end{abstract}

\section{Introduction}

Surgical and catheter ablation of atrial fibrillation (AF) have emerged as a safe ${ }^{1}$ and effective alternative to antiarrhythmic drug therapy ${ }^{2-4,5,67,8,9,10}$. The perceived success rate of these procedures is highly dependent on the duration of AF recurrence that defines treatment failure as well as the intensity and duration of monitoring ${ }^{11-14}$. Historically, clinical trials have used varying cutoffs of AF recurrence with varying methods to detect the endpoint. Society guidelines define AF recurrence as any AF, atrial tachycardia (AT) or atrial flutter (AFL) of at least 30 seconds after a 90 -day blanking period ${ }^{15}$, a definition that has traditionally been tested with intermittent ECGs and Holter monitors performed at fixed intervals or with symptoms. With the rapid expansion of direct to consumer monitoring technologies, the ease of patient reported self-monitoring of AF recurrences post-intervention has already started to shift the paradigm towards a more patient- facing approach.

\section{Indications for monitoring}

AF monitoring following ablation and surgery is if often used to assess efficacy of treatment, particularly in the setting of clinical trials, as well as to guide clinical management ${ }^{16}$. Monitoring allows correlation of symptoms with recurrent arrhythmias as many patients may have symptoms during normal sinus rhythm or from other arrhythmias apart from AF. In addition, monitoring allows one to screen for drug induced proarrhythmia, evaluate efficacy of rate control during recurrent AF and aid in decision making surrounding anticoagulation in low-risk patients.

\section{Definition of success}

Procedural success varies substantially depending on how success is defined. The first 90 days following ablation, termed the blanking period, are characterized by a high burden of atrial arrhythmias resulting from 
atrial inflammation which does not necessarily portend a reduction in long term success and is frequently not used clinically or in research studies to indicate treatment failure. While one 30 second episode of AF over the course of a year, excluding the blanking period, technically constitutes treatment failure to maintain sinus rhythm, this low burden of AF is arguably clinically irrelevant to the individual patient and does not increase stroke risk ${ }^{17}$. AF burden reduction ${ }^{18}$ and improvement in quality of life $(\mathrm{QoL})^{7}$ may be more clinically relevant endpoints. Clinical trials such as CIRCA Dose used insertable cardiac monitors (ICMs) for AF monitoring post-ablation and showed a reduction in AF burden by $99 \%$ even though almost half had one or more AF recurrences at 12 months ${ }^{18}$. Using a real-world dataset of 665 patients with a cardiac implantable electronic device (CIED) having undergone an AF catheter ablation, we showed that AF free survival at 1 year post-blanking period for patients with paroxysmal AF varied from 28.2 to $72.1 \%$ for $\mathrm{AF}$ events $>6$ min and $>24$ hrs, respectively, despite a $99.6 \%$ reduction in AF burden, and AF free survival at 1 year for patients with persistent AF varied from 24.9 to $60 \%$ for AF events $>6$ min and $>7$ days with $>23 \mathrm{hrs}$, respectively, despite a $99.3 \%$ reduction in AF burden (Figure 1) ${ }^{19}$. These findings were similar to those found in the LINQ AF study in which 419 patients that had an ICM implanted following AF catheter ablation had a success rate that varied from 46 to $79 \%$, depending on what threshold was used to define success.

A rational approach to $\mathrm{AF}$ detection post-intervention should rest in part on the minimal duration of $\mathrm{AF}$ associated with major events, with stroke constituting the most feared consequence of the disease. While AF burden has been directly correlated with stroke risk ${ }^{20-23}$, the exact duration of AF that is required to increase the risk of stroke is still being debated. In a sample of 725 patients with implanted pacemakers, Capucci and colleagues showed that the risk of stroke was increased with AF episodes $>24 \mathrm{hrs}^{24}$. The TRENDS study ${ }^{25}$, which subsequently enrolled 2486 patients with CIEDs, found that only AF episodes $>5.5$ hours over a 30day rolling window were associated with an increased risk of thromboembolic events (HR 2.2). The initial analysis of the ASSERT trial, which enrolled 2580 patients with CIEDs, found that AF events $>6$ minutes in duration increased the risk of stroke or systemic embolization (HR 2.49) ${ }^{26}$. However, a subsequent analysis of the ASSERT data showed that only AF events $>24$ hours were associated with an increased stroke risk (HR 3.24) and that patients with $<24 \mathrm{hrs}$ of $\mathrm{AF}$ had the same stroke risk as those without $\mathrm{AF}^{27}$. What further complicates the issue is the observation that the association between AF duration and stroke may be dependent on the individuals underlying risk factors. A study of 21768 non-anticoagulated patients with CIEDs demonstrated a clear interaction between $\mathrm{AF}$ duration and $\mathrm{CHA}_{2} \mathrm{DS}_{2}$-VASc score, such that patients with a $\mathrm{CHA}_{2} \mathrm{DS}_{2}$-VASc score of 2 required $>23.5 \mathrm{hrs}$ of $\mathrm{AF}$ but those with a $\mathrm{CHA}_{2} \mathrm{DS}_{2}$-VASc score of 4 required only $>6 \mathrm{~min}$ of $\mathrm{AF}$ to have a stroke risk $>1 \%$, the threshold some suggest is the "tipping point" for anticoagulation $^{20}$.

\section{Conventional monitoring devices}

Historically, post-intervention AF monitoring has been performed with either periodic electrocardiograms (ECGs), external ambulatory ECG monitors ${ }^{16}$ or CIEDs when present. External ambulatory ECG monitors include continuous Holter monitors that record all ECG data, event monitors that record patient events when manually triggered, loop recorders that continuously monitor the patient's rhythm and store events that are either patient triggered or automatically triggered by abnormal rhythms and real-time ambulatory telemetry that sends continuous ECG recordings to a central monitoring station where technicians can alert physicians in real time to events. CIEDs capable of monitor AF recurrence and burden include ICMs as well as pacemakers and implantable cardiac defibrillators with an atrial lead or with atrial sensing poles on the ventricular lead.

Clinical practice guidelines recommend that for routine clinical care, patients should have, at a minimum, an ECG at each follow up visit, including at 3 months and then every 6 months for 2 years, with more intense monitoring at the discretion of the provider. For the purposes of clinical trials, guidelines recommend, in addition to ECGs at each follow-up visit, a minimum of a $24 \mathrm{hr}$ Holter at the end of the follow-up period (for paroxysmal AF) or every 6 months (for persistent AF) and event monitoring done regularly and at the time of symptoms from the end of the 3 months blanking period to end of follow-up (for paroxysmal AF) or 
symptom driven event monitoring (for persistent AF).

Not surprisingly, the longer and more frequently you monitor for AF, the more frequently it is discovered. This challenge is well illustrated by the concept of AF density where intermittent monitoring has a low sensitivity for detecting AF in individuals with infrequent episodes of AF concentrated over short timeframes and are therefore less likely to have $\mathrm{AF}$ detected with infrequent, intermittent monitoring. ${ }^{20}$ Consistent with this observation, a meta-analysis of $66 \mathrm{AF}$ ablation studies including a total of 6941 patients showed that the method of $\mathrm{AF}$ detection was predictive of procedural success rather than any specifics of the ablation procedure itself, with studies that used CIEDs to monitor for AF recurrence showing the highest $\mathrm{AF}$ recurrence rates. Using a monitoring strategy simulation in 665 patients with CIEDs undergoing an AF ablation, we have shown that commonly used strategies of external ambulatory monitoring have relatively low sensitivity and negative predictive value in detecting post-ablation AF, with a commonly used strategy of a single 7 -day continuous Holter monitor recording having $<50 \%$ sensitivity in detecting AF recurrence $>6$ min at 1 year $(\text { Figure } 2)^{19}$.

\section{The emergence of personal monitoring devices}

In recent years there has been an increasing array of direct-to-consumer devices capable of detecting AF, predominantly utilizing smart phones and smart watches. Survey data from the Pew Research Center (www.pewresearch.org) suggests that the proportion of US adults with a smart phone has roughly doubled since 2011 to over $80 \%$ and the proportion who use a smartwatch or fitness tracker now exceeds $20 \%$, with ongoing expansion each year as more products enter the marketplace. The resulting growth in patient driven heart rhythm monitoring without direct physician oversight has empowered patients to investigate their own rhythm abnormalities either as a screening tool or for disease management. Current methods available to monitor for AF include smart phone finger pulse wave photoplethysmography (PPG) using downloadable applications and hardware already present in modern smartphones, external electrodes that communicate with an app downloaded to a smart phone used to generate an mobile single or six-lead electrocardiogram (iECG), or smart watch PPG with or without single lead iECG confirmation (by touching the crown of the watch with opposite hand to create a single lead ECG).

Personal monitoring devices have been shown to have a relatively high accuracy in detecting AF. The SEARCH-AF study used iECGs to screen 1000 pharmacy customers for AF using 12 lead ECG confirmation, finding a sensitivity of $98.5 \%$ and specificity of $91.4 \%$ for AF, diagnosing new AF in $1.5 \%$ of customers, all of which had a CHADS-VASc score of 2 or more and therefore would conceivably benefit from anticoagulation to reduce stroke risk ${ }^{28,29}$. Chan and colleagues screened 1013 primary care patients with a smartphone camera-based PPG algorithm against iECG tracings reviewed by two cardiologists and found a sensitivity of $92.9 \%$ and specificity of $97.7 \%$, though lower sensitivities are reported outside the research setting ${ }^{30}$. The WATCH AF trial ${ }^{31}$ compared the accuracy of an automated wrist watch PPG algorithm in diagnosing AF in 672 hospitalized patients as compared to a single lead iECG interpreted by cardiologists as a reference. Although $21.8 \%$ of PPG datasets were not interpretable, the remaining datasets had a sensitivity of $93.7 \%$ and specificity of $98.2 \%$ for detecting AF. Contact-free facial PPG using subtle beat-to-beat variations of skin color has similarly shown promise in detecting AF with similar sensitivity ${ }^{32}$. As a proof of concept on a larger community scale, the Apple Heart Study recruited 419,297 participants with smart watches using an irregular pulse notification algorithm and mailed a 7-day continuous Holter monitor patch to those with an irregular pulse. They found that $0.52 \%$ of participants received notifications of an irregular pulse, of which $34 \%$ had AF diagnosed on the monitoring patch that was placed on average 13 days later ${ }^{33}$. Of the 86 participants that had an irregular pulse notification subsequently while wearing a patch, the positive predictive value of the irregular pulse notification was 0.84 in detecting AF confirmed on the patch.

Advancements in wearable AF-sensors raises the possibility that assessment of AF duration and burden over long time horizons will no longer require implantable devices. In a study of 24 patients Wasserlauf and colleagues compared the accuracy of a simultaneous deep convolutional neural network PPG algorithm with single lead iECG confirmation using KardiaBand ${ }^{\mathrm{TM}}$ (AliveCor, Mountain View, CA) with simultaneous ICM recordings ${ }^{34}$. The smart watch algorithm had a sensitivity of $97.5 \%$ in detecting AF episodes $>1$ hour and 
had a high correlation $\left(\mathrm{R}^{2}\right.$ 0.996) with the duration of AF recorded on the ICM. With studies suggesting that stroke risk is highest for the 30 days following an episode of $\mathrm{AF}^{35}$ and that hours and not minutes of $\mathrm{AF}$ is likely necessary to increase stroke risk in those with few vascular risk factors, wearable AF-detection devices raise the possibility that stroke risk and bleeding risk can be reduced by targeted, time-delimited, "pill-in-pocket" anticoagulation in those with infrequent episodes of paroxysmal AF, either spontaneously or as the result of AF ablation or surgery.

\section{Conclusion}

AF monitoring following ablation is an indispensable tool to guide patient management. The traditional strategy of intermittent external ambulatory monitoring devices to assess recurrence of AF as a binary outcome is increasingly being recognized as inaccurate and misses the opportunity for tailored patient management. AF burden and duration assessment by personal monitoring devices, in the form of direct to consumer smart watches and smart phones, holds promise to guide individual patient management and is likely to empower patients to manage their own disease.

\section{Acknowledgements}

None.

\section{Authorship Contributions}

All Authors contributed equally to the preparation of the manuscript.

\section{Disclosure of Conflicts of Interest}

Rod Passman is a consultant to Medtronic and Abbott, receives research support from Pfizer and receives royalties from UpToDate. Graham Lohrmann has no disclosures.

\section{References}

1. Packer DL, Mark DB, Robb RA et al. Effect of Catheter Ablation vs Antiarrhythmic Drug Therapy on Mortality, Stroke, Bleeding, and Cardiac Arrest among Patients with Atrial Fibrillation: The CABANA Randomized Clinical Trial. In: JAMA - Journal of the American Medical Association. Vol 321. American Medical Association; 2019:1261-1274. doi:10.1001/jama.2019.0693

2. Packer DL, Kowal RC, Wheelan KR et al. Cryoballoon ablation of pulmonary veins for paroxysmal atrial fibrillation: First results of the North American arctic front (STOP AF) pivotal trial. J Am Coll Cardiol . 2013;61(16):1713-1723. doi:10.1016/j.jacc.2012.11.064

3. Steven D, Rostock T, Lutomsky B et al. What is the real atrial fibrillation burden after catheter ablation of atrial fibrillation? A prospective rhythm analysis in pacemaker patients with continuous atrial monitoring. Eur Heart J . 2008;29(8):1037-1042. doi:10.1093/eurheartj/ehn024

4. Nielsen JC, Johannessen A, Raatikainen P et al. Long-term efficacy of catheter ablation as first-line therapy for paroxysmal atrial fibrillation: 5-year outcome in a randomised clinical trial.Heart . 2017;103(5):368376. doi:10.1136/heartjnl-2016-309781

5. Andrade JG, Wells GA, Deyell MW et al. Cryoablation or Drug Therapy for Initial Treatment of Atrial Fibrillation. $N$ Engl J Med . Published online November 16, 2020:NEJMoa2029980. doi:10.1056/NEJMoa2029980

6. Kirchhof P, Camm AJ, Goette A et al. Early Rhythm-Control Therapy in Patients with Atrial Fibrillation. N Engl J Med . 2020;383(14):1305-1316. doi:10.1056/nejmoa2019422

7. Mark DB, Anstrom KJ, Sheng S et al. Effect of Catheter Ablation vs Medical Therapy on Quality of Life among Patients with Atrial Fibrillation: The CABANA Randomized Clinical Trial. In: JAMA Journal of the American Medical Association. Vol 321. American Medical Association; 2019:1275-1285. doi:10.1001/jama.2019.0692 
8. Blomström-Lundqvist C, Gizurarson S, Schwieler J et al. Effect of Catheter Ablation vs Antiarrhythmic Medication on Quality of Life in Patients with Atrial Fibrillation: The CAPTAF Randomized Clinical Trial.JAMA - J Am Med Assoc . 2019;321(11):1059-1068. doi:10.1001/jama.2019.0335

9. Marrouche NF, Brachmann J, Andresen D et al. Catheter ablation for atrial fibrillation with heart failure. N Engl J Med . 2018;378(5):417-427. doi:10.1056/NEJMoa1707855

10. Di Biase L, Mohanty P, Mohanty S et al. Ablation Versus Amiodarone for Treatment of Persistent Atrial Fibrillation in Patients With Congestive Heart Failure and an Implanted Device: Results From the AATAC Multicenter Randomized Trial. In: Circulation. Vol 133. Lippincott Williams and Wilkins; 2016:1637-1644. doi:10.1161/CIRCULATIONAHA.115.019406

11. Wechselberger S, Kronborg M, Huo Y et al. Continuous monitoring after atrial fibrillation ablation: the LINQ AF study. EP Eur . 2018;20(FI_3):f312-f320. doi:10.1093/europace/euy038

12. Dinshaw L, Schäffer B, Akbulak Ö et al. Long-term efficacy and safety of radiofrequency catheter ablation of atrial fibrillation in patients with cardiac implantable electronic devices and transvenous leads. $J$ Cardiovasc Electrophysiol . 2019;30(5):679-687. doi:10.1111/jce.13890

13. Heeger CH, Tscholl V, Salloum O et al. What is the real recurrence rate after cryoballoon-based pulmonary vein isolation? Lessons from rhythm follow-up based on implanted cardiac devices with continuous atrial monitoring. Hear Rhythm . 2018;15(12):1844-1850. doi:10.1016/j.hrthm.2018.07.016

14. Ferreira-Martins J, Howard J, Al-Khayatt B et al. Outcomes of paroxysmal atrial fibrillation ablation studies are affected more by study design and patient mix than ablation technique. $J$ Cardiovasc Electrophysiol . 2018;29(11):1471-1479. doi:10.1111/jce.13745

15. Calkins H, Kuck KH, Cappato R et al. 2012 HRS/EHRA/ECAS Expert Consensus Statement on Catheter and Surgical Ablation of Atrial Fibrillation: Recommendations for Patient Selection, Procedural Techniques, Patient Management and Follow-up, Definitions, Endpoints, and Research Trial Design. Hear Rhythm . 2012;9(4):632-696.e21. doi:10.1016/j.hrthm.2011.12.016

16. Mittal S, Movsowitz C, Steinberg JS. Ambulatory external electrocardiographic monitoring: Focus on atrial fibrillation. J Am Coll Cardiol . 2011;58(17):1741-1749. doi:10.1016/j.jacc.2011.07.026

17. Swiryn S, Orlov M V., Benditt DG et al. Clinical Implications of Brief Device-Detected Atrial Tachyarrhythmias in a Cardiac Rhythm Management Device Population: Results from the Registry of Atrial Tachycardia and Atrial Fibrillation Episodes. Circulation . 2016;134(16):1130-1140. doi:10.1161/CIRCULATIONAHA.115.020252

18. Andrade JG, Champagne J, Dubuc M et al. Cryoballoon or Radiofrequency Ablation for Atrial Fibrillation Assessed by Continuous Monitoring: A Randomized Clinical Trial. Circulation . 2019;140(22):17791788. doi:10.1161/CIRCULATIONAHA.119.042622

19. Lohrmann G, Kaplan R, Ziegler PD, Monteiro J, Passman R. Atrial fibrillation ablation success defined by duration of recurrence on cardiac implantable electronic devices. J Cardiovasc Electrophysiol . Published online October 27, 2020:jce.14781. doi:10.1111/jce.14781

20. Kaplan RM, Koehler J, Ziegler PD, Sarkar S, Zweibel S, Passman RS. Stroke risk as a function of atrial fibrillation duration and CHA2DS2-VASc score. Circulation . 2019;140(20):1639-1646. doi:10.1161/CIRCULATIONAHA.119.041303

21. Healey JS, Connolly SJ, Gold MR et al. Subclinical Atrial Fibrillation and the Risk of Stroke. $N$ Engl J Med . 2012;366(2):120-129. doi:10.1056/NEJMoa1105575

22. Glotzer T V., Daoud EG, Wyse DG et al. The Relationship between daily atrial tachyarrhythmia burden from implantable device diagnostics and stroke risk the trends study. Circ Arrhythmia Electrophysiol . 2009;2(5):474-480. doi:10.1161/CIRCEP.109.849638 
23. Steinhubl SR, Waalen J, Edwards AM et al. Effect of a home-Based wearable continuous ECG monitoring patch on detection of undiagnosed atrial fibrillation the mSToPS randomized clinical trial. JAMA - J Am Med Assoc . 2018;320(2):146-155. doi:10.1001/jama.2018.8102

24. Capucci A, Santini M, Padeletti L et al. Monitored atrial fibrillation duration predicts arterial embolic events in patients suffering from bradycardia and atrial fibrillation implanted with antitachycardia pacemakers. J Am Coll Cardiol . 2005;46(10):1913-1920. doi:10.1016/j.jacc.2005.07.044

25. Glotzer T V., Daoud EG, Wyse DG et al. The Relationship between daily atrial tachyarrhythmia burden from implantable device diagnostics and stroke risk the trends study. Circ Arrhythmia Electrophysiol . 2009;2(5):474-480. doi:10.1161/CIRCEP.109.849638

26. Healey JS, Connolly SJ, Gold MR et al. Subclinical Atrial Fibrillation and the Risk of Stroke. $N$ Engl $J$ Med . 2012;366(2):120-129. doi:10.1056/nejmoa1105575

27. Van Gelder IC, Healey JS, Crijns HJGM et al. Duration of device-detected subclinical atrial fibrillation and occurrence of stroke in ASSERT. Eur Heart J . 2017;38(17):1339-1344. doi:10.1093/eurheartj/ehx042

28. Lowres N, Neubeck L, Salkeld G et al. Feasibility and cost-effectiveness of stroke prevention through community screening for atrial fibrillation using iPhone ECG in pharmacies: The SEARCH-AF study. Thromb Haemost . 2014;111(6):1167-1176. doi:10.1160/TH14-03-0231

29. Lau JK, Lowres N, Neubeck L et al. IPhone ECG application for community screening to detect silent atrial fibrillation: A novel technology to prevent stroke. Int J Cardiol . 2013;165(1):193-194. doi:10.1016/j.ijcard.2013.01.220

30. Chan PH, Wong CK, Poh YC et al. Diagnostic Performance of a Smartphone-Based Photoplethysmographic Application for Atrial Fibrillation Screening in a Primary Care Setting. J Am Heart Assoc . 2016;5(7). doi:10.1161/JAHA.116.003428

31. Dorr M, Nohturfft V, Brasier N et al. The WATCH AF Trial: SmartWATCHes for Detection of Atrial Fibrillation. JACC Clin Electrophysiol . 2019;5(2):199-208. doi:10.1016/j.jacep.2018.10.006

32. Yan BP, Lai WHS, Chan CKY et al. Contact-free screening of atrial fibrillation by a smartphone using facial pulsatile photoplethysmographic signals. J Am Heart Assoc . 2018;7(8). doi:10.1161/JAHA.118.008585

33. Perez M V., Mahaffey KW, Hedlin H et al. Large-Scale Assessment of a Smartwatch to Identify Atrial Fibrillation. N Engl J Med . 2019;381(20):1909-1917. doi:10.1056/nejmoa1901183

34. Wasserlauf J, You C, Patel R, Valys A, Albert D, Passman R. Smartwatch Performance for the Detection and Quantification of Atrial Fibrillation. Circ Arrhythmia Electrophysiol . 2019;12(6). doi:10.1161/CIRCEP.118.006834

35. Turakhia MP, Ziegler PD, Schmitt SK et al. Atrial Fibrillation Burden and Short-Term Risk of Stroke: Case-Crossover Analysis of Continuously Recorded Heart Rhythm from Cardiac Electronic Implanted Devices. Circ Arrhythmia Electrophysiol . 2015;8(5):1040-1047. doi:10.1161/CIRCEP.114.003057

\section{Figures:}

\section{Hosted file}

image1.emf available at https://authorea.com/users/352241/articles/497578-the-futureof-long-term-monitoring-following-catheter-and-surgical-intervention-for-atrialfibrillation

Figure 1. Freedom from Atrial Fibrillation (AF) Following Catheter Ablation Stratified by Event Definition in Patients with Paroxysmal AF (Panel A, $\mathrm{n}=248$ ) and Persistent AF (Panel B, $\mathrm{n}=417$ ). 


\section{AF: Atrial fibrillation}

\section{Hosted file}

image2.emf available at https://authorea.com/users/352241/articles/497578-the-futureof-long-term-monitoring-following-catheter-and-surgical-intervention-for-atrialfibrillation

Figure 2. Estimated Sensitivity and NPV by Monitoring Strategy at 1 Year Post Blanking Period in Paroxysmal (Panel A, $\mathrm{n}=137$ ) and Persistent AF (Panel B, $\mathrm{n}=232$ ) Patients.

AF: Atrial fibrillation; NPV: Negative predictive value 